\title{
Genomic variants in Fas-mediated apoptosis pathway predict a poor response to Platinum-based Chemo- therapy for Chinese Gastric Cancer Patients
}

\author{
Tingting Zhao ${ }^{1^{*}}$, Wei Li $^{*}$, Jinfei chen ${ }^{3 凶}$ and Weisong Qin ${ }^{1 凶}$ \\ 1. National Clinical Research Center of Kidney Diseases, Jinling Hospital, Nanjing University School of Medicine, Nanjing, 21000, China. \\ 2. Department of Gynecology, Zhenjiang Maternity and Childcare Hospital, Zhenjiang, 212000, China. \\ 3. Cancer Center, Taikang Xianlin Drum Tower Hospital, Nanjing University School of Medicine, Nanjing, 21000, China. \\ *These authors contributed equally to this work.
}

$\triangle$ Corresponding authors: Jinfei chen, Cancer Center, Taikang Xianlin Drum Tower Hospital, Nanjing University School of Medicine, Nanjing 210046, China. E-mail: jinfeichen@sohu.com; Weisong Qin, National Clinical Research Center of Kidney Diseases, Jinling Hospital, Nanjing University School of Medicine, Nanjing, 21000, China. E-mail: qinweisong@163.com.

(C) The author(s). This is an open access article distributed under the terms of the Creative Commons Attribution License (https://creativecommons.org/licenses/by/4.0/). See http://ivyspring.com/terms for full terms and conditions.

Received: 2020.01.27; Accepted: 2020.02.09; Published: 2021.01.01

\begin{abstract}
Platinum-based adjuvant chemotherapy is very common for gastric cancer (GC) patients, but the chemotherapy sensitivity is very heterogeneous. The genomic variants and the gene-gene interactions involved in Fas-mediated apoptosis pathway including Fas (FAS $1377 \mathrm{G}>\mathrm{A}$ and $670 \mathrm{~A}>\mathrm{G}$ ), FasL (FASL $844 \mathrm{C}>\mathrm{T}$ ) and caspase-8 (CASP8 $-6526 \mathrm{~N}$ ins > del or I > D), may paly vital roles in the response to platinum-based treatment. In our investigation, 662 stage II-III postoperative GC patients were enrolled between 1998 and 2006. 261 patients accepted platinum-based regimens and the remaining 401 were not. The log rank tests, Kaplan Meier plots, Pearson chi-square tests, Student t-tests and Cox regression analyses were performed. For the chemotherapy cohort, FAS $1377 \mathrm{G}>$ A or FAS 670 A > G variants alone was related with inferior survival, and a greater than additive effect was identified when patients simultaneously carrying FAS 1377 GA and FAS 670 GA genotypes. But the poor response was neutralized when patients simultaneously carrying FASL 844 C > T or CASP8 $-6526 \mathrm{~N}$ ins > del mutations. Our study suggested that FAS $1377 \mathrm{G}>\mathrm{A}$ and FAS $670 \mathrm{~A}>\mathrm{G}$ variants may serve as potential biomarkers to predict the response to platinum-based adjuvant chemotherapy, and the gene-gene interactions involved in Fas-mediated apoptosis pathway may enhance or neutralize the chemosensitivity.
\end{abstract}

Key words: Fas-mediated apoptosis, gastric cancer (GC), genetic variants, chemotherapy

\section{Introduction}

In spite of the mortality is decreasing in the past few decades, gastric cancer (GC) still represents the second most common cancer-related deaths all over the word, particularly in developing countries [1-3]. Surgery is the main treatment for GC patients, but two thirds of GC patients recur after curative resection [4]. So, postoperative chemotherapy is globally considered as the standard treatment in clinic [5]. The 5 -year survival rate for the cancer patients accepted postoperative chemotherapy extends from $49.6 \%$ to $55.3 \%$ compared with tumor resection alone [6]. Up to now, although novel therapeutic strategies have been developed rapidly, platinum-based chemotherapy remains the best choice for GC patients [7]. But the clinical therapy benefits are various mainly due to the differences in chemosensitivity. Genetic variants involved in Fas-mediated apoptosis pathway may exert crucial roles in the treatment effect for platinum-based adjuvant chemotherapy $[8,9]$. Thus, identifications of the single-nucleotide polymorphisms (SNPs) and the gene-gene interactions involved in Fas-mediated apoptosis pathway are of great importance to make more precise evaluation of the chemotherapy efficacy and then design personalized therapy. 
Apoptosis, also named programmed cell death (PCD), is relate to diverse biological processes, such as the maintenance of tissues homeostasis, the development of cell proliferation, the disorder of immune surveillance, and the elimination of cancer cells [10,11]. And the abnormal regulations of apoptosis can lead to numerous human diseases, including cancer $[12,13]$. As we all known, apoptosis mainly occurs through two signaling pathways, including the extrinsic pathway and the intrinsic pathway. Fas/FasL system triggered extrinsic pathway is a main mechanism for the induction of cell and tissue apoptosis [14]. Fas (APO-1TNFSF6/CD95) interacts with FasL (CD95L) to initiate the death signal cascade and consequentially actives caspase-8 (CASP8), eventually leading to cell death $[15,16]$. The activations of Fas-induced apoptosis may exert essential functions in the treatment of platinum-based chemotherapy, and the dysregulations of the Fas/FasL signaling pathway might affect the chemosensitivity [17,18]. The decreased Fas level might down-regulate of Fas-induced apoptosis, while the elevated FASL level would enhance the ability of cancer cells to fight against immune system by killing FAS sensitive lymphocytes, which might subsequently affect chemotherapeutic response and change the prognosis of patients accepted chemotherapy $[19,20]$.

FAS gene is located on chromosome 10q24.1, which is a cell-surface receptor of tumor necrosis factor (TNF) receptor superfamily [21]. FAS is widely expressed in various tissues and interacts with FASL to active apoptotic signaling pathway [22]. FASL gene (another TNF receptors), mapped on chromosome 1q23, is mainly expressed in cytotoxic T lymphocytes and natural killer cells [23]. CASP8 gene, situated in chromosome 2q33-34, is the key regulator of the FAS/FASL-induced apoptosis in T lymphocytes. And CASP8 may exert critical roles in the defense mechanisms against hyper-proliferation and tumorigenesis [24].

Recently, some novel SNPs in the promoter regions of FASL, FAS and CASP8 have been identified. The FAS $670 \mathrm{~A}>\mathrm{G}(\mathrm{rs} 1800682 \mathrm{~A}>\mathrm{G})$ and 1377 G > A (rs2234767 G > A) variants could disrupt Sp1 (Transcription factor Sp1) and STAT1 (Signal transducer and activator of transcription 1) transcription factor binding sites, which may reduce the level of Fas gene and diminish its promoter activity [25]. In regard to FASL, the transition from C to $\mathrm{T}$ at position $844($ rs763110 $\mathrm{C}>\mathrm{T}$ ) creates a new binding motif for another transcription factor, CAAT/enhancer-binding protein $\beta$, which may lead to obvious reduction of FASL [26]. As for CASP8, a six-nucleotide deletion variant of AGTAAG at position $652(-6526 \mathrm{~N}$ ins $>$ del or I > D, rs3834129) in the promoter region may destroy the $\mathrm{Sp} 1$ transcriptional activator binding element [27]. So, CASP8 $-6526 \mathrm{~N}$ ins $>$ del genetic polymorphism could decrease CASP8 mRNA expression and diminish the activity of CASP8, which may significantly reduce T lymphocytes apoptosis and obviously improve immune surveillance [28].

Previous investigations demonstrated that those functional genetic variations of Fas/FasL/CASP8 may influence the susceptibility to gastric cancer or affected the sensitivity to platinum-based chemotherapy for some types of cancer, including non-small-cell lung cancer, malignant pleural mesothelioma and so on [29-31]. However, the associations between genomic variants involved in Fas-mediated apoptosis pathway, with the response to platinum-based chemotherapy for gastric cancer patients, was still unclear. In addition, whether the interactions between the genes could enhance or neutralize the sensitivity to platinum-based treatment was also confused. So, for the first time, we systematically investigated the individual and synergistic effects of the variants of FASL, FAS and CASP8 on the response to platinum-based adjuvant chemotherapy for postoperative stage II-III GC patients on the basis of large amount of clinical data.

\section{Methods}

\section{Enrolled patients}

The retrospective cohort study was approved by the Institutional Review Committee of Nanjing Medical University (Nanjing, China). Patients enrolled in our investigation must meet the following criteria: a) histopathological and imaging diagnosed as stage II-III GC; b) no radiotherapy or chemotherapy before surgery; c) the clinical data were complete without missing; d) signed an informed consent to use postoperative tissues for clinical researches; e) conducted operation at the Yixing People's Hospital (Yixing, Jiangsu Province, China) from January 1998 to December 2006; f) accepted follow-up after surgery. Above all, a total of 662 stage II-III GC patients were collected, and the patients' general clinical and pathologic characteristics were summarized in Table 1. Within one month after operation, 261 recruited patients accepted platinum-based chemotherapy, but the other 401 had not due to different reasons. Chemotherapy was given when the patients reached the following level: neutrophil counts $\geq 1.5 \times 10^{9} / \mathrm{L}$, hemoglobin level $\geq 8 \mathrm{~g} / \mathrm{dl}$, platelet counts $\geq 100 \times$ $10^{9} / \mathrm{L}$, as well as no symptom of organ toxicities. If necessary, Antiemetics and mannitol diuresis were used. The follow-up period was counted from the 
operation date to the death or last follow-up time (March 31, 2009, up to 119.0 months), and the median follow-up time was 68.5 months. The TNM staging were assessed on the basis of the TNM classification of the American Joint Committee on Cancer (7th edition). The histopathology of GC was classified into diffuse and intestinal types on the basis of Lauren's Criterion [32]. And all of our researches were based on the clinical guidelines of Nanjing Medical University.

\section{Genotyping}

The methods that extract genomic DNA from paraffin sections were similar to previous description [33]. The above genes variants were measured by SNaPshot using an ABI fluorescence-based assay allelic identification method or automated sequencing. Supplementary Table S4 listed the primer sequence of each SNP. The polymorphisms were determined using an ABI3130 genetic analyzer and the Genemapper version 4.0 was adopted to analyze genotypes (Applied Biosystems, Forster City, CA) [34]. Two people randomly selected $10 \%$ of the samples to independently verify the genotype the results were $100 \%$ consistent.

\section{Statistical analysis}

When the median survival time (MST) cannot be got, we chose mean survival time instead. Student t-tests or the Pearson chi-square tests was adopted on the basis of the variable type to elucidate the correlations between each and/or combined genotypes with clinicopathologic parameters. Kaplan-Meier plots and log-rank tests were calculated by SPSS 20.0 software (Inc, Chicago, IL, USA). The estimation of the crude/adjusted hazard ratios (HRs) and $95 \%$ confidence intervals (95\% CIs) were calculated by univariate or multivariate Cox regression analysis. All of the above tests were bilateral and $P<0.05$ was defined as statistical significance.

\section{Results}

\section{Clinical features of the study subjects}

Table 1 showed the clinical features of the 662 recruited GC patients in II-III phase. The median age was 61.0 years (range 28 to 83 years). During the total number of 119.0 months follow-up, 335 patients died. 261 patients received platinum-based adjuvant chemotherapy, and the other 401 did not. For the cohort did not accept platinum-based treatment, the overall survival time was closely related to histological type, tumor differentiation, invasion depth, Lymph node metastasis and TNM stage $(P<$ $0.05)$. In particular, the mortality rate of no-chemotherapy patients with diffuse-type (MST, 37 months) was $54.5 \%$ notably higher $(\mathrm{HR}=1.545,95 \% \mathrm{CI}=$ 1.1125-2.122, $P=0.006$ ) than that of patients with intestinal type (MST, 74 months). Compared with patients with poor differentiation (MST, 41 months) and mucinous or signet-ring cell (MST, 32 months), patients with well differentiated (MST, 70 months) had $36.9 \%(\mathrm{HR}=1.369,95 \% \mathrm{CI}=1.000-1.875)$ and $116.5 \%(\mathrm{HR}=2.165,95 \% \mathrm{CI}=1.224-3.829, P=0.015)$ reduced death risk, respectively. Patients with T3/T4 invasion depth (MST, 48 months) had 64.7\% higher death risk $(\mathrm{HR}=1.647,95 \% \mathrm{CI}=1.002-2.706, P=$ 0.045) than those with T1/T2 invasion depth (MST, 75 months). Patients with N1/N2/N3 lymph node metastasis (MST, 41 months) had 46.5\% higher death risk $(\mathrm{HR}=1.465,95 \% \mathrm{CI}=1.032-2.080, P=0.030)$ than those with no lymph node metastasis (MST, 67 months). Meanwhile, Patients in TNM stage III (MST, 37 months) had $91.7 \%$ higher death risk $(\mathrm{HR}=1.917$, 95\% CI $=1.394-2.637, P<0.001)$ when compared with those patients in stage II (MST, 75 months). However, no similar higher death risk was identified in the chemotherapy cohort.

Table 1. Clinical characteristics of the gastric cancer patients in two cohorts

\begin{tabular}{|c|c|c|c|c|c|c|c|c|}
\hline \multirow[t]{2}{*}{ Variable } & \multicolumn{4}{|c|}{ Chemotherapy (261) } & \multicolumn{4}{|c|}{ No chemotherapy (401) } \\
\hline & Patients/deaths & MST (mouths) & $P$ & $\mathrm{HR}(95 \% \mathrm{CI})^{\mathrm{a}}$ & Patients/deaths & MST (mouths) & $P$ & $\mathrm{HR}(95 \% \mathrm{CI})^{\mathrm{a}}$ \\
\hline \multicolumn{9}{|c|}{ Age (years) } \\
\hline$\leq 60$ & $149 / 65$ & $62^{\mathrm{b}}$ & 0.335 & 1 & $162 / 88$ & 48 & 0.606 & 1 \\
\hline$>60$ & $112 / 53$ & 50 & & $1.194(0.830-1.717)$ & $238 / 128$ & 50 & & $1.074(0.817-1.412)$ \\
\hline \multicolumn{9}{|l|}{ Sex } \\
\hline Male & $212 / 96$ & 62 & 0.977 & 1 & $305 / 161$ & 53 & 0.440 & 1 \\
\hline Female & $49 / 22$ & 55 & & $1.007(0.633-1.601)$ & $96 / 56$ & 38 & & $1.126(0.831-1.527)$ \\
\hline \multicolumn{9}{|c|}{ Tumor size } \\
\hline$\leq 5$ & $143 / 61$ & $63^{b}$ & 0.269 & 1 & 212/109 & 59 & 0.130 & 1 \\
\hline$>5$ & $118 / 57$ & 51 & & $1.224(0.857-1.756)$ & $189 / 108$ & 43 & & $1.226(0.939-1.601)$ \\
\hline \multicolumn{9}{|c|}{ Tumor site } \\
\hline No-cardia & $163 / 76$ & 50 & 0.433 & 1 & $256 / 142$ & 41 & 0.430 & 1 \\
\hline Cardia & $98 / 42$ & $62^{b}$ & & $0.861(0.591-1.255)$ & $145 / 75$ & 59 & & $0.894(0.676-1.183)$ \\
\hline \multicolumn{9}{|c|}{ Histological type } \\
\hline Intestinal & $75 / 37$ & 62 & 0.333 & 1 & $123 / 50$ & 74 & 0.006 & 1 \\
\hline
\end{tabular}




\begin{tabular}{|c|c|c|c|c|c|c|c|c|}
\hline \multirow[t]{2}{*}{ Variable } & \multicolumn{4}{|c|}{ Chemotherapy (261) } & \multicolumn{4}{|c|}{ No chemotherapy (401) } \\
\hline & Patients/deaths & MST (mouths) & $P$ & $\operatorname{HR}(95 \% \mathrm{CI})^{\mathrm{a}}$ & Patients/deaths & MST (mouths) & $P$ & $\operatorname{HR}(95 \% \mathrm{CI})^{\mathrm{a}}$ \\
\hline Diffuse & $186 / 81$ & 55 & & $1.100(0.905-1.336)$ & $278 / 167$ & 37 & & $1.545(1.125-2.122)$ \\
\hline \multicolumn{9}{|l|}{ Tumor differentiation } \\
\hline Well & $96 / 46$ & 62 & 0.512 & 1 & $133 / 56$ & 70 & 0.015 & 1 \\
\hline Poorly & $145 / 63$ & $61^{\mathrm{b}}$ & & $0.804(0.549-1.176)$ & $231 / 134$ & 41 & & $1.369(1.000-1.875)$ \\
\hline Mucinous or signet-ring cell & $16 / 7$ & 52 & & $0.813(0.367-1.802)$ & $21 / 15$ & 32 & & $2.165(1.224-3.829)$ \\
\hline \multicolumn{9}{|l|}{ Depth of invasion } \\
\hline $\mathrm{T} 1 / \mathrm{T} 2$ & $25 / 15$ & 29 & 0.108 & 1 & $43 / 17$ & $75^{b}$ & 0.045 & 1 \\
\hline T3/T4 & $236 / 103$ & $61^{\mathrm{b}}$ & & $0.645(0.375-1.110)$ & $353 / 196$ & 48 & & $1.647(1.002-2.706)$ \\
\hline \multicolumn{9}{|l|}{ Lymph node metastasis } \\
\hline NO & $54 / 19$ & $69 \mathrm{~b}$ & 0.057 & 1 & $89 / 38$ & 67 & 0.03 & 1 \\
\hline N1/N2/N3 & $207 / 99$ & 51 & & $1.599(0.978-2.614)$ & $309 / 177$ & 41 & & $1.465(1.032-2.080)$ \\
\hline \multicolumn{9}{|l|}{ TNM stage } \\
\hline II & $75 / 32$ & 62 & 0.430 & 1 & $127 / 49$ & $75^{b}$ & $<0.001$ & 1 \\
\hline III & $186 / 86$ & 55 & & $1.176(0.783-1.765)$ & $274 / 168$ & 37 & & $1.917(1.394-2.637)$ \\
\hline
\end{tabular}

a: Adjusted for age and sex;

b: Mean survival time was provided when MST could not be calculated;

c: Information was not available for two patients;

HR, hazard ratio; CI, confidence interval; MST, median survival time.

Table 2. Associations of FAS, FASL and Casp8 polymorphisms with gastric cancer patients' survival in both cohorts

\begin{tabular}{|c|c|c|c|c|c|c|c|c|c|}
\hline \multirow[t]{2}{*}{ Genetic model } & \multirow[t]{2}{*}{ Genotypes } & \multicolumn{4}{|l|}{ Chemotherapy } & \multicolumn{4}{|l|}{ No chemotherapy } \\
\hline & & Patients/deaths & MST (mouths) & $P$ & $\mathrm{HR}(95 \% \mathrm{CI})^{\mathrm{a}}$ & Patients/Deaths & MST (mouths) & $P$ & $\mathrm{HR}(95 \% \mathrm{CI})^{\mathrm{a}}$ \\
\hline \multicolumn{10}{|c|}{ FAS rs22347671377 G>A } \\
\hline \multirow[t]{3}{*}{ Codominant model } & GG & $113 / 40$ & $69 \mathrm{~b}$ & 0.007 & 1 & $147 / 78$ & 48 & 0.973 & 1 \\
\hline & GA & $115 / 60$ & 50 & & $1.734(1.161-2.589)$ & 198/108 & 50 & & $1.020(0.762-1.365)$ \\
\hline & AA & $26 / 15$ & 29 & & $2.135(1.176-3.875)$ & $38 / 19$ & 88 & & $0.966(0.585-1.596)$ \\
\hline Dominant model & GA/AA & $141 / 75$ & 51 & 0.002 & $1.801(1.225-2.648)$ & $236 / 127$ & 50 & 0.936 & $1.012(0.763-1.341)$ \\
\hline \multirow[t]{2}{*}{ Recessive model } & GG/GA & $228 / 100$ & $61^{\mathrm{b}}$ & 0.091 & 1 & $345 / 186$ & 50 & 0.849 & 1 \\
\hline & AA & $26 / 15$ & 29 & & $1.586(0.920-2.734)$ & $38 / 19$ & 88 & & $0.955(0.596-1.532)$ \\
\hline \multicolumn{10}{|c|}{ FAS $r s 1800682670 A>G$} \\
\hline \multirow[t]{3}{*}{ Codominant model } & AA & $94 / 34$ & $68^{\mathrm{b}}$ & 0.036 & 1 & $120 / 66$ & 48 & 0.460 & 1 \\
\hline & GA & $121 / 62$ & 43 & & $1.702(1.119-2.590)$ & $214 / 117$ & 47 & & $1.008(0.745-1.364)$ \\
\hline & GG & $36 / 18$ & 37 & & $1.591(0.898-2.821)$ & $41 / 18$ & 62 & & $0.742(0.440-1.249)$ \\
\hline Dominant model & GA/GG & $157 / 80$ & 43 & 0.010 & $1.676(1.120-2.507)$ & $255 / 135$ & 53 & 0.794 & $0.962(0.716-1.291)$ \\
\hline \multirow[t]{2}{*}{ Recessive model } & $\mathrm{AA} / \mathrm{GA}$ & $215 / 96$ & 62 & 0.547 & 1 & 334183 & 48 & 0.213 & 1 \\
\hline & GG & $36 / 18$ & 37 & & $1.166(0.704-1.929)$ & $41 / 18$ & 52 & & $0.738(0.454-1.198)$ \\
\hline \multicolumn{10}{|c|}{ FASL $r s 763110844 C>T$} \\
\hline \multirow[t]{3}{*}{ Codominant model } & $\mathrm{CC}$ & $70 / 31$ & $51^{\mathrm{b}}$ & 0.536 & 1 & $90 / 39$ & 77 & 0.418 & 1 \\
\hline & $\mathrm{TC}$ & $144 / 70$ & 52 & & $1.011(0.656-1.559)$ & $234 / 134$ & 48 & & $1.248(0.873-1.784)$ \\
\hline & TT & $31 / 13$ & $54^{\mathrm{b}}$ & & $0.471(0.116-1.918)$ & $43 / 23$ & 43 & & $1.065(0.635-1.783)$ \\
\hline Dominant model & $\mathrm{TC} / \mathrm{TT}$ & $175 / 83$ & 55 & 0.442 & $0.851(0.561-1.290)$ & $277 / 157$ & 47 & 0.269 & $0.987(0.732-1.333)$ \\
\hline \multirow[t]{2}{*}{ Recessive model } & $\mathrm{CC} / \mathrm{TC}$ & 214/101 & 52 & 0.333 & 1 & $324 / 173$ & 53 & 0.635 & 1 \\
\hline & TT & $31 / 13$ & $54^{\mathrm{b}}$ & & $0.754(0.432-1.344)$ & $43 / 23$ & 43 & & $0.901(0.583-1.392)$ \\
\hline \multicolumn{10}{|c|}{ CASP8 $-6526 \mathrm{~N}$ ins $>$ del } \\
\hline \multirow[t]{3}{*}{ Codominant model } & II & $153 / 71$ & 52 & 0.492 & 1 & $226 / 132$ & 36 & 0.008 & 1 \\
\hline & DI & $82 / 38$ & $52^{\mathrm{b}}$ & & $1.037(0.699-1.538)$ & $143 / 70$ & 63 & & $0.741(0.554-0.991)$ \\
\hline & DD & $13 / 4$ & $72^{\mathrm{b}}$ & & $0.564(0.206-1.543)$ & $12 / 2$ & $100^{\mathrm{b}}$ & & $0.202(0.054-0.815)$ \\
\hline & DI/DD & $95 / 42$ & $61^{b}$ & 0.832 & $0.960(0.655-1.406)$ & $155 / 72$ & 65 & 0.011 & $0.690(0.518-0.921)$ \\
\hline \multirow[t]{2}{*}{ Recessive model } & II/DI & $235 / 109$ & 55 & 0.239 & 1 & $369 / 202$ & 48 & 0.021 & 1 \\
\hline & DD & $13 / 4$ & $72^{\mathrm{b}}$ & & $0.557(0.205-1.510)$ & $12 / 2$ & $100^{\mathrm{b}}$ & & $0.227(0.056-0.913)$ \\
\hline
\end{tabular}

a: Adjusted for age and sex;

b: Mean survival time was provided when MST could not be calculated;

I, a six-nucleotide insertion; D; a six-nucleotide deletion.

\section{FAS $1377 \mathrm{G}>\mathrm{A}$ and FAS $670 \mathrm{~A}>\mathrm{G}$ variants contributed to a poor response to platinum- based chemotherapy}

We applied the Cox regression analyses in various genetic models to assess the associations between the genetic variants involved in the Fasinduced apoptosis pathway with the overall survival among the two cohorts (Table 2). For the patients underwent chemotherapy, significantly reduced survival time were observed for FAS 1377 G > A and
$670 \mathrm{~A}>\mathrm{G}$ mutations in codominant and dominant models.

Our results demonstrated that chemotherapy patients with FAS 1377 GA + AA genotypes had $80.1 \%$ significantly higher death risk than those carrying GG genotype $(\mathrm{HR}=1.801,95 \% \mathrm{CI}=$ 1.225-2.648, $P=0.002$, Table 2, Figure 1A). The MST of 1377 GA + AA genotypes was shortened from 69 to 51 months when compared with GG genotype. Similarly, chemotherapy patients with FAS 670 GA + GG 
genotypes also exhibited $67.6 \%$ obviously higher death risk than those carrying $\mathrm{AA}$ genotype $(\mathrm{HR}=$ $1.676,95 \% \mathrm{CI}=1.120-2.507, P=0.010$, Table 2, Figure 1B). Compared with AA genotype, the MST of 670 GA + GG genotypes were shortened from 68 to 43 months.

\section{Prolongation of no chemotherapy patients' survival time with CASP8 -652 6NI > D variant}

As for CASP8 $-6526 \mathrm{~N} \mathrm{I}>\mathrm{D}$ variants, we observed that, in all genetic models, CASP8 -652 6N I $>\mathrm{D}$ mutation obviously protected untreated GC patients from death in all genetic models. No chemotherapy patients with 6526 DI + DD genotypes exhibited obviously reduced death risk by $31 \%(\mathrm{HR}=$ $0.690,95 \% \mathrm{CI}=0.518-0.921, P=0.010$, Table 2) compared with those carrying II genotype. The MST in patients carrying DI + DD genotypes was prolonged from 36 to 65 months when compared with those with II genotype. In addition, untreated GC patients with codominant or recessive models also showed remarkably longer survival time.

No significant relationships were identified between other polymorphisms including CASP8 -652 $6 \mathrm{~N} \mathrm{I}>\mathrm{D}$ and FASL $844 \mathrm{C}>\mathrm{T}$ with the survival of GC patients accepted platinum-based treatment in any genetic models. Furthermore, none of the remaining three genes polymorphisms except CASP8 -652 6N I > $\mathrm{D}$ were associated with the overall survival for the no chemotherapy patients.

\section{Stratified analyses of FAS 670 A > G and 1377 $G>A$ variants on the increased susceptibility to chemoresistance}

To better explore the associations between FAS $670 \mathrm{~A}>\mathrm{G}$ and $1377 \mathrm{G}>\mathrm{A}$ variants with the overall survival of patients received chemotherapy, stratified analysis was performed based on the ages, tumor sizes and sites, tumor differentiation, histological types, lymph node metastasis, invasion depth, metastasis distant and TNM stage (Table 3). For FAS 1377 G > A variant, compared with the GG genotype, the FAS 1377 GA + AA genotypes were obviously associated with poor survival for chemotherapy patients in age $\leq 60(\mathrm{HR}=1.981,95 \% \mathrm{CI}=1.181-3.323$, $P=0.008)$, tumor size $>5 \mathrm{~cm}(\mathrm{HR}=2.056,95 \% \mathrm{CI}=$ 1.134-3.726, $P=0.014)$, tumor arising at Non-cardia $(\mathrm{HR}=2.011,95 \% \mathrm{CI}=1.220-3.314, P=0.005)$, tumor of diffuse type $(\mathrm{HR}=1.791,95 \% \mathrm{CI}=1.118-2.869, P=$ $0.013)$, poorly differentiated tumor $(\mathrm{HR}=1.805,95 \%$ $\mathrm{CI}=1.064-3.060, P=0.025)$, stage $\mathrm{T} 3 / \mathrm{T} 4$ invasion depth $(\mathrm{HR}=1.851,95 \% \mathrm{CI}=1.225-2.795, P=0.003)$, N1/N2/N3 lymph node metastasis (HR $=1.678,95 \%$ $\mathrm{CI}=1.108-2.541, P=0.013)$, no distant metastasis (HR $=1.801,95 \% \mathrm{CI}=1.225-2.646, P=0.002)$ and $\mathrm{TNM}$ stage III $(\mathrm{HR}=1.707,95 \% \mathrm{CI}=1.092-2.670, P=0.017$, Table 3, Figure $2 \mathrm{~A}_{1} \& \mathbf{2} \mathrm{A}_{2}$ ).

Similarly, stratification analysis for FAS 670 A > $G$ polymorphism also showed that GA + GG genotypes had distinct relationships with the inferior survival for patients with age $\leq 60(\mathrm{HR}=1.852,95 \% \mathrm{CI}$ $=1.076-3.188, P=0.032)$, tumor arising at non-cardia ( $\mathrm{HR}=1.806,95 \% \mathrm{CI}=1.067-3.055, P=0.024)$, tumor of intestinal type $(\mathrm{HR}=2.475,95 \% \mathrm{CI}=1.192-5.140, P=$ $0.011)$, well to moderately differentiation $(\mathrm{HR}=2.087$, $95 \% \mathrm{CI}=1.092-3.989, \mathrm{P}=0.022)$, stage $\mathrm{T} 3 / \mathrm{T} 4$ invasion depth $(\mathrm{HR}=1.715,95 \% \mathrm{CI}=1.115-2.637, P=0.012)$, and N1/N2/N3 lymph node metastasis $(\mathrm{HR}=1.537$, $95 \% \mathrm{CI}=1.00-2.372, P=0.048$, Table 3, Figure $2 \mathbf{B}_{1} \&$ 2B $\mathbf{B}_{2}$.
A

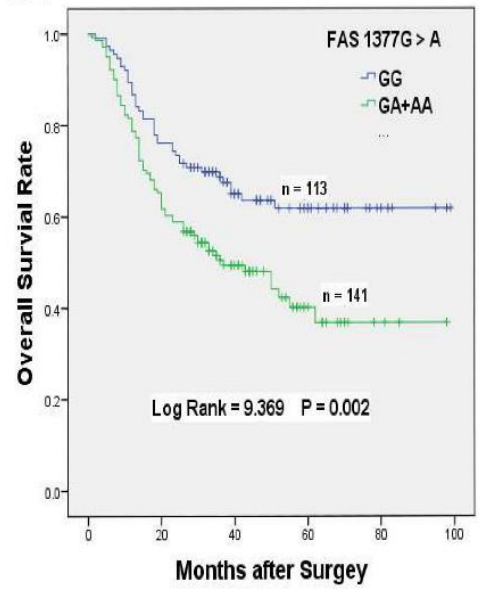

B

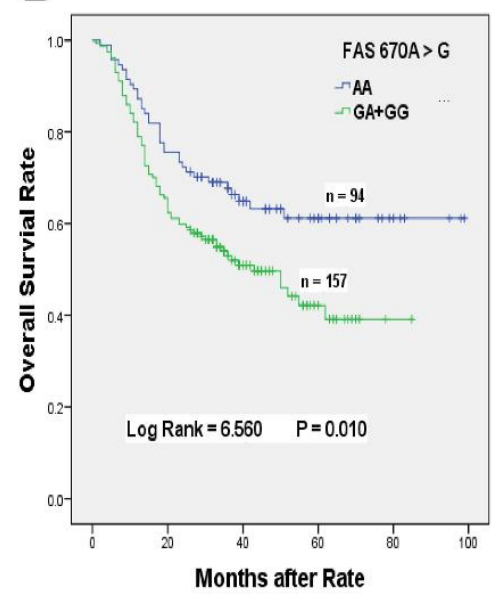

$\mathrm{C}$

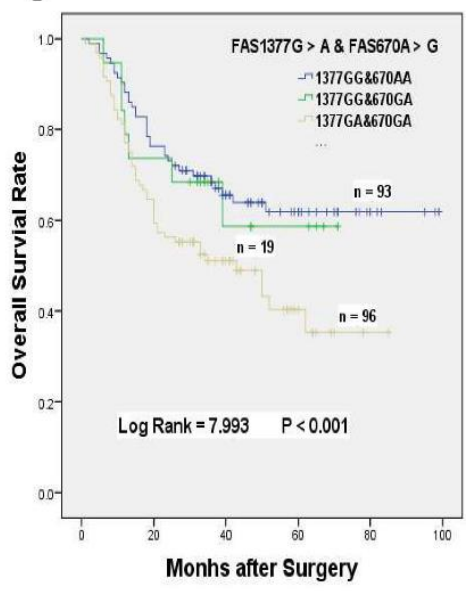

Figure 1. Overall survival of FAS $1377 \mathrm{G}>\mathrm{A}$ and/or $670 \mathrm{~A}>\mathrm{G}$ polymorphisms for $\mathrm{GC}$ patients accepted platinum-based chemotherapy. (A) Kaplan-Meier survival curve of $F A S$ $1377 \mathrm{G}>\mathrm{A}$ in dominant genotypes for overall survival of GC patients received chemotherapy. (B) Kaplan-Meier survival curve of FAS 670A > G in dominant genotypes for overall survival of GC patients received chemotherapy. (C) Kaplan-Meier survival curve of FAS $1377 \mathrm{G}>\mathrm{A}$ interaction with $670 \mathrm{~A}>\mathrm{G}$ in heterozygous genotypes for overall survival of GC patients received chemotherapy. 
A1

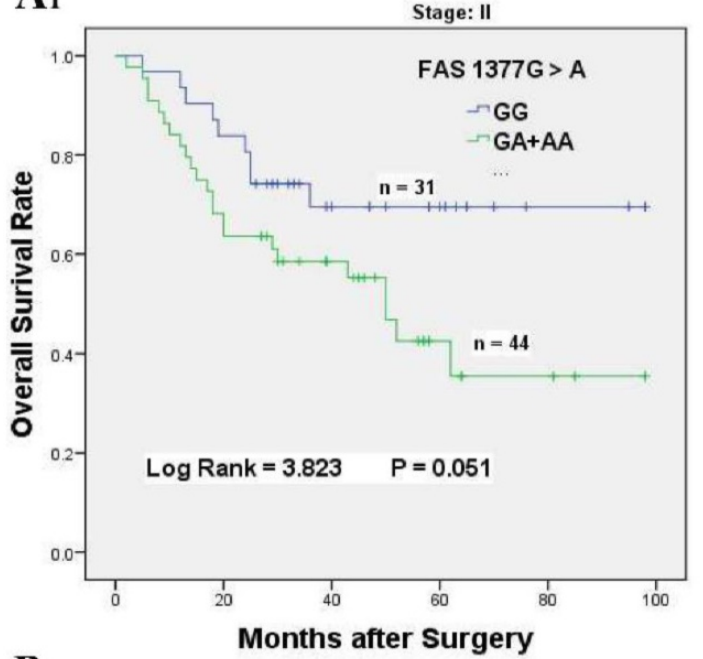

B1

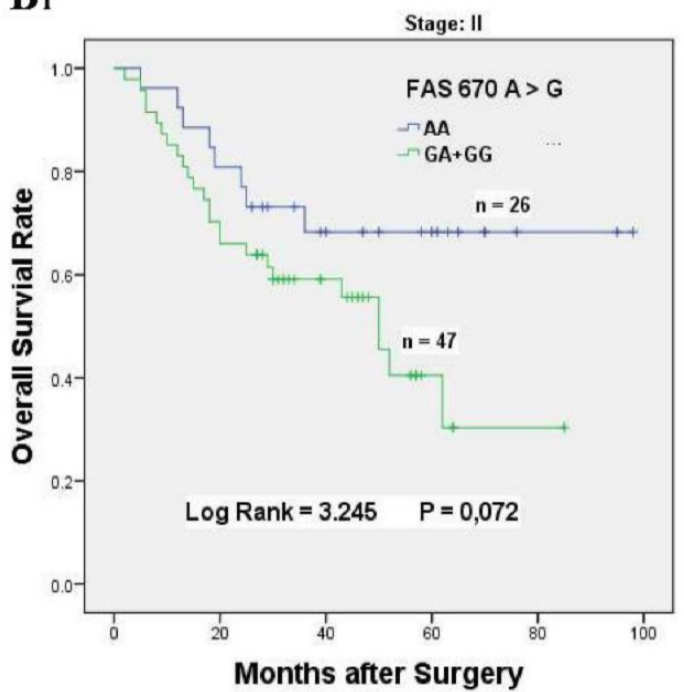

A2

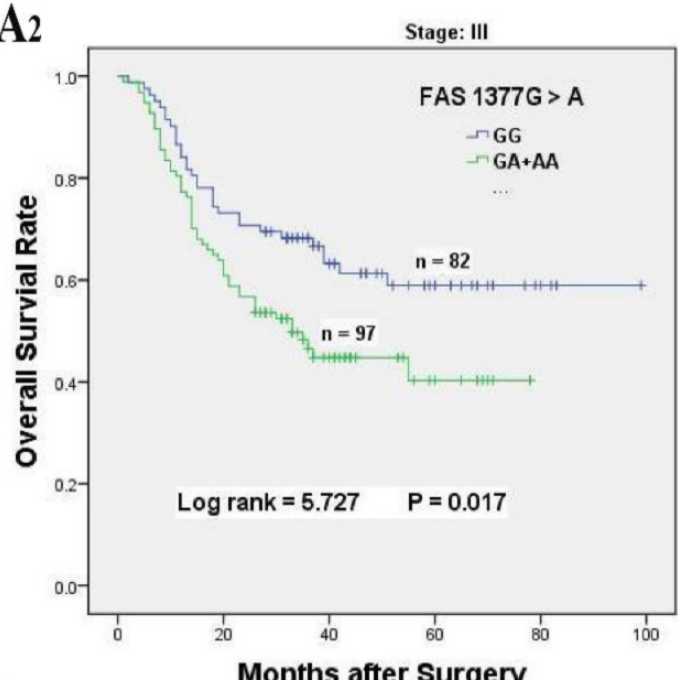

$\mathbf{B}_{2}$

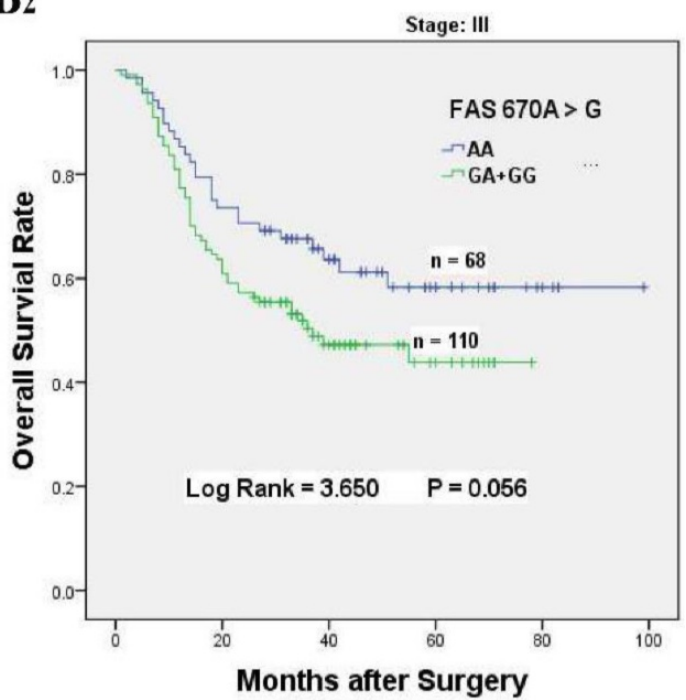

Figure 2. Kaplan-Meier survival curves of FAS $1377 \mathrm{G}>A$ and $670 \mathrm{~A}>\mathrm{G}$ for overall survival of stage II-III GC patients accepted platinum-based chemotherapy. (A) Overall survival of $F A S 1377 G>A$ in dominant genotypes for stage II $\left(A_{1}\right)$ and stage III $\left(A_{2}\right)$ chemotherapy patients, respectively. (B) Overall survival of $F A S 670 A>G$ in dominant genotypes for stage II $\left(B_{1}\right)$ and stage III $\left(B_{2}\right)$ chemotherapy patients, respectively.

Table 3. Stratified analysis of the FAS $1377 \mathrm{G}>\mathrm{A}$ and $670 \mathrm{~A}>\mathrm{G}$ polymorphisms with gastric cancers' overall survival for the patients received platinum-based chemotherapy

\begin{tabular}{|c|c|c|c|c|c|c|c|c|}
\hline \multirow[t]{2}{*}{ Variables } & \multicolumn{2}{|c|}{ FAS 1377 G > A Genotypes } & \multirow[t]{2}{*}{$\operatorname{HR}(95 \% \mathrm{CI})^{\mathrm{a}}$} & \multirow[t]{2}{*}{$P$} & \multicolumn{2}{|c|}{ FAS 670 A > G Genotypes } & \multirow[t]{2}{*}{$\operatorname{HR}(95 \% \mathrm{CI})^{\mathrm{a}}$} & \multirow[t]{2}{*}{$P$} \\
\hline & GG & GA/AA & & & $\mathrm{AA}$ & GA/GG & & \\
\hline Total & $113 / 40$ & $141 / 75$ & $1.801(1.225-2.648)$ & 0.002 & $94 / 34$ & $157 / 80$ & $1.676(1.120-2.507)$ & 0.010 \\
\hline \multicolumn{9}{|l|}{ Age } \\
\hline$\leq 60$ & $67 / 22$ & $79 / 64$ & $1.981(1.181-3.323)$ & 0.008 & $57 / 19$ & $85 / 43$ & $1.852(1.076-3.188)$ & 0.032 \\
\hline$>60$ & $46 / 18$ & $62 / 33$ & $1.558(0.876-2.773)$ & 0.124 & $37 / 15$ & $72 / 37$ & $1.441(0.790-2.628)$ & 0.266 \\
\hline \multicolumn{9}{|l|}{ Tumor size } \\
\hline$\leq 5 \mathrm{~cm}$ & $69 / 25$ & $71 / 35$ & $1.570(0.938-2.628)$ & 0.081 & $59 / 21$ & $80 / 39$ & $1.614(0.946-2.752)$ & 0.074 \\
\hline$>5 \mathrm{~cm}$ & $44 / 15$ & $70 / 40$ & $2.056(1.134-3.726)$ & 0.014 & $35 / 13$ & $77 / 41$ & $1.672(0.895-3.123)$ & 0.099 \\
\hline \multicolumn{9}{|l|}{ Tumor site } \\
\hline Non-cardia & $66 / 22$ & $92 / 52$ & $2.011(1.220-3.314)$ & 0.005 & $56 / 19$ & $100 / 53$ & $1.806(1.067-3.055)$ & 0.024 \\
\hline Cardia & $47 / 18$ & $49 / 23$ & $1.477(0.795-2.745)$ & 0.211 & $38 / 15$ & $57 / 27$ & $1.469(0.779-2.770)$ & 0.229 \\
\hline \multicolumn{9}{|l|}{ Histological type } \\
\hline Intestinal type & $38 / 14$ & $36 / 22$ & $1.944(0.993-3.805)$ & 0.047 & $31 / 10$ & $43 / 27$ & $2.475(1.192-5.140)$ & 0.011 \\
\hline Diffuse type & $75 / 26$ & $105 / 53$ & $1.791(1.118-2.869)$ & 0.013 & $63 / 24$ & $114 / 53$ & $1.406(0.867-2.280)$ & 0.162 \\
\hline \multicolumn{9}{|l|}{ Tumor differentiation } \\
\hline Well to moderately & $46 / 17$ & $49 / 28$ & $1.761(0.962-3.223)$ & 0.061 & $38 / 13$ & $57 / 33$ & $2.087(1.092-3.989)$ & 0.022 \\
\hline Poor & $61 / 21$ & $81 / 41$ & $1.805(1.064-3.060)$ & 0.025 & $51 / 19$ & $88 / 41$ & $1.407(0.816-2.425)$ & 0.214 \\
\hline Mucinous/signet-ring cell & $5 / 1$ & $11 / 6$ & $3.682(0.441-30.715)$ & 0.197 & $4 / 1$ & $12 / 6$ & $2.965(0.352-24.94)$ & 0.295 \\
\hline \multicolumn{9}{|l|}{ Depth of invasion } \\
\hline $\mathrm{T} 1 / \mathrm{T} 2$ & $9 / 5$ & $16 / 10$ & $1.182(0.403-3.470)$ & 0.758 & $7 / 4$ & $18 / 11$ & $1.076(0.342-3.388)$ & 0.899 \\
\hline
\end{tabular}




\begin{tabular}{|c|c|c|c|c|c|c|c|c|}
\hline \multirow[t]{2}{*}{ Variables } & \multicolumn{2}{|c|}{ FAS 1377 G > A Genotypes } & \multirow[t]{2}{*}{$\operatorname{HR}(95 \% \mathrm{CI})^{\mathrm{a}}$} & \multirow[t]{2}{*}{$P$} & \multicolumn{2}{|c|}{ FAS 670 A > G Genotypes } & \multirow[t]{2}{*}{$\operatorname{HR}(95 \% \mathrm{CI})^{\mathrm{a}}$} & \multirow[t]{2}{*}{$P$} \\
\hline & GG & GA/AA & & & AA & GA/GG & & \\
\hline T3/T4 & $104 / 35$ & $125 / 65$ & $1.851(1.225-2.795)$ & 0.003 & $87 / 30$ & $139 / 69$ & $1.715(1.115-2.637)$ & 0.012 \\
\hline \multicolumn{9}{|c|}{ Lymph node metastasis } \\
\hline N0 & $22 / 4$ & $32 / 15$ & $3.020(1.001-9.110)$ & 0.038 & $19 / 4$ & $33 / 15$ & $2.735(0.902-2.372)$ & 0.063 \\
\hline N1/N2/N3 & $91 / 36$ & $109 / 60$ & $1.678(1.108-2.541)$ & 0.013 & $75 / 30$ & $124 / 65$ & 1.537 (1.00-2.372) & 0.048 \\
\hline \multicolumn{9}{|c|}{ Distant metastasis } \\
\hline M0 & $113 / 40$ & $141 / 75$ & $1.801(1.225-2.646)$ & 0.002 & $94 / 34$ & $157 / 80$ & $1.676(1.12-2.507)$ & 0.010 \\
\hline \multicolumn{9}{|l|}{ TNM stage } \\
\hline II & $31 / 9$ & $44 / 23$ & $1.073(0.517-2.226)$ & 0.051 & $26 / 8$ & $47 / 24$ & $2.056(0.919-4.60)$ & 0.072 \\
\hline III & $82 / 31$ & $97 / 52$ & $1.707(1.092-2.670)$ & 0.017 & $68 / 26$ & $110 / 56$ & $1.564(0.981-2.49)$ & 0.056 \\
\hline
\end{tabular}

a: Adjusted for age and sex;

b: Heterogeneity test for differences between groups;

c: Information was not available for two patients.

Table 4. The effects of the gene-gene interactions on the survival of gastric cancer patients received platinum-based chemotherapy

\begin{tabular}{|c|c|c|c|c|c|}
\hline Combined genotypes & Patients & Deaths & SST (months) & $P$ & $\mathrm{HR}(95 \% \mathrm{CI})^{\mathrm{a}}$ \\
\hline \multicolumn{6}{|c|}{ FAS $1377 G>A$ and FAS $670 A>G$} \\
\hline $\begin{array}{l}\text { FAS } 1377 G G+F A S \\
670 A A\end{array}$ & 93 & 33 & $69^{\mathrm{b}}$ & \multirow[t]{4}{*}{$<0.001$} & 1 \\
\hline $\begin{array}{l}\text { FAS } 1377 G G+F A S \\
670 G A\end{array}$ & 19 & 7 & $50^{\mathrm{b}}$ & & $1.128(0.499-2.552)$ \\
\hline $\begin{array}{l}\text { FAS 1377GA + FAS } \\
670 A A\end{array}$ & 1 & 1 & 5 & & $40.84(4.925-338.6)$ \\
\hline $\begin{array}{l}\text { FAS 1377GA + FAS } \\
670 \text { GA }\end{array}$ & 96 & 51 & 43 & & $1.830(1.179-2.840)$ \\
\hline \multicolumn{6}{|c|}{ AS $1377 G>A$ and FASL $844 C>T$} \\
\hline $\begin{array}{l}\text { FAS } 1377 G G+F A S L \\
844 C C\end{array}$ & 30 & 11 & $57 \mathrm{~b}$ & \multirow[t]{4}{*}{0.012} & 1 \\
\hline $\begin{array}{l}\text { FAS } 1377 G G+F A S L \\
844 T C+T T\end{array}$ & 79 & 28 & 70 & & $0.734(0.364-1.480)$ \\
\hline $\begin{array}{l}\text { FAS } 1377 G A+A A+ \\
\text { FASL } 844 C C\end{array}$ & 40 & 20 & 23 & & $1.568(0.751-3.274)$ \\
\hline $\begin{array}{l}\text { FAS } 1377 G A+A A+ \\
\text { FASL } 844 T C+T T\end{array}$ & 94 & 53 & 36 & & $1.449(0.765-2.777)$ \\
\hline \multicolumn{6}{|c|}{ FAS $1377 G>A$ and CASP8 6526 ins $>$ del } \\
\hline $\begin{array}{l}\text { FAS } 1377 \text { GG + } \\
\text { CASP8 } 6526 \text { II }\end{array}$ & 73 & 26 & $68^{\mathrm{b}}$ & \multirow[t]{4}{*}{0.037} & 1 \\
\hline $\begin{array}{l}\text { FAS } 1377 \text { GG + } \\
\text { CASP8 } 6526 \text { DI }\end{array}$ & 32 & 12 & $56^{\mathrm{b}}$ & & $1.036(0.523-2.053)$ \\
\hline $\begin{array}{l}\text { FAS } 1377 \text { GA + } \\
\text { CASP8 } 6526 \text { II }\end{array}$ & 66 & 38 & 43 & & $1.918(1.164-3.162)$ \\
\hline $\begin{array}{l}\text { FAS } 1377 \text { GA + } \\
\text { CASP8 } 6526 \text { DI }\end{array}$ & 39 & 19 & 33 & & $1.627(0.899-2.945)$ \\
\hline \multicolumn{6}{|c|}{ FAS $670 A>G$ and FASL $844 C>T$} \\
\hline $\begin{array}{l}\text { FAS } 670 A A+F A S L \\
844 C C\end{array}$ & 23 & 9 & $55^{\mathrm{b}}$ & \multirow[t]{4}{*}{0.185} & 1 \\
\hline $\begin{array}{l}\text { FAS 670AA+FASL } \\
\text { 844TC }\end{array}$ & 56 & 21 & $68^{\mathrm{b}}$ & & $0.778(0.355-1.705)$ \\
\hline $\begin{array}{l}\text { FAS 670GA + FASL } \\
844 C C\end{array}$ & 34 & 14 & $32^{\mathrm{b}}$ & & $1.156(0.500-2.673)$ \\
\hline $\begin{array}{l}\text { FAS 670GA+ FASL } \\
844 T C\end{array}$ & 69 & 39 & 35 & & $1.384(0.670-2.860)$ \\
\hline \multicolumn{6}{|c|}{ FAS $670 A>G$ and CASP8 6526 ins $>$ del } \\
\hline $\begin{array}{l}\text { FAS 670AA + CASP8 } \\
6526 \text { II }\end{array}$ & 65 & 23 & $68^{\mathrm{b}}$ & \multirow[t]{4}{*}{0.059} & 1 \\
\hline $\begin{array}{l}\text { FAS } 670 A A+C A S P 8 \\
6526 D D+D I\end{array}$ & 27 & 10 & $70^{\mathrm{b}}$ & & $0.984(0.468-2.068)$ \\
\hline $\begin{array}{l}\text { FAS } 670 \text { GA+GG + } \\
\text { CASP8 } 6526 \text { II }\end{array}$ & 87 & 47 & 43 & & $1.790(1.086-2.950)$ \\
\hline $\begin{array}{l}\text { FAS } 670 \text { GA+GG }+ \\
\text { CASP8 } 6526 D D+D I\end{array}$ & 65 & 31 & 37 & & $1.630(0.948-2.801)$ \\
\hline
\end{tabular}

\section{The gene-gene interactions influenced the overall survival of GC patients accepted platinum-based adjuvant chemotherapy}

In consideration of the complex interactions between the above genes, we focused on the effects of the gene-gene interactions on the prognosis of GC patients underwent platinum-based treatment (Supplementary Table S1-S3). In univariate analysis, FAS 670 A > G or $1377 \mathrm{G}>$ A variants alone was related to inferior survival, and a greater than additive effect was identified when patients simultaneously carrying FAS 1377 GA and FAS 670 GA genotypes $(\mathrm{HR}=1.830,95 \% \mathrm{CI}=1.179-2.840$, log-rank $P<0.001$, Table 4, Figure 1C). Whereas, the poor survival of patients carrying FAS 1377 GA + AA or FAS $670 \mathrm{GA}$ genotypes disappeared when GC patients simultaneous with FASL $844 \mathrm{TC}+\mathrm{TT}$ or FASL 844 TC genotypes, which were not related to the survival of GC patients accepted treatment in univariate analysis $(\mathrm{HR}=1.449,95 \% \mathrm{CI}=0.765-2.777$, $P=0.012 ; \mathrm{HR}=1.384,95 \% \mathrm{CI}=0.670-2.860, P=0.185$, respectively, Table 4, Figure $\mathbf{3} \mathbf{A}_{\mathbf{1}} \& \mathbf{3} \mathbf{A}_{\mathbf{2}}$ ). Meanwhile, the inferior survival of FAS 1377 GA were maintained when patients simultaneously carrying CASP8 -652 $6 \mathrm{~N}$ II genotype $(\mathrm{HR}=1.918,95 \% \mathrm{CI}=1.164-3.162)$, but the shortened survival time vanished when patients also carrying CASP8 $-6526 \mathrm{~N}$ DI genotype $(\mathrm{HR}=$ $1.627,95 \% \mathrm{CI}=0.899-2.945, P=0.037$, Table 4, Figure $\mathbf{3 B}_{1}$ ). The similar results were existed when patients simultaneously carrying FAS 670 GA + GG and CASP8 $-6526 \mathrm{~N}$ II or CASP8 $-6526 \mathrm{~N}$ DD + DI genotypes $(\mathrm{HR}=1.790,95 \% \mathrm{CI}=1.086-2.950$ and $\mathrm{HR}=$ 1. $630,95 \% \mathrm{CI}=0.948-2.801, P=0.059$ respectively, Table 4, Figure $\mathbf{3 B}_{2}$ ).

\section{Discussion}

Our present research systematically explored the clinical significances of the SNPs related to the Fas-induced apoptosis pathway and the gene-gene interactions on the prognosis of GC patients in II-III stage that underwent platinum-based adjuvant chemotherapy. Our investigations demonstrated that FAS 670 A > G and $1377 \mathrm{G}>$ A mutations contributed to a poor response to platinum-based treatment, and the treatment efficacy was also interfered by gene-gene interactions. So, our investigation provided the useful basics to make more precise evaluation of the chemotherapy efficacy and then further design personalized therapy. 

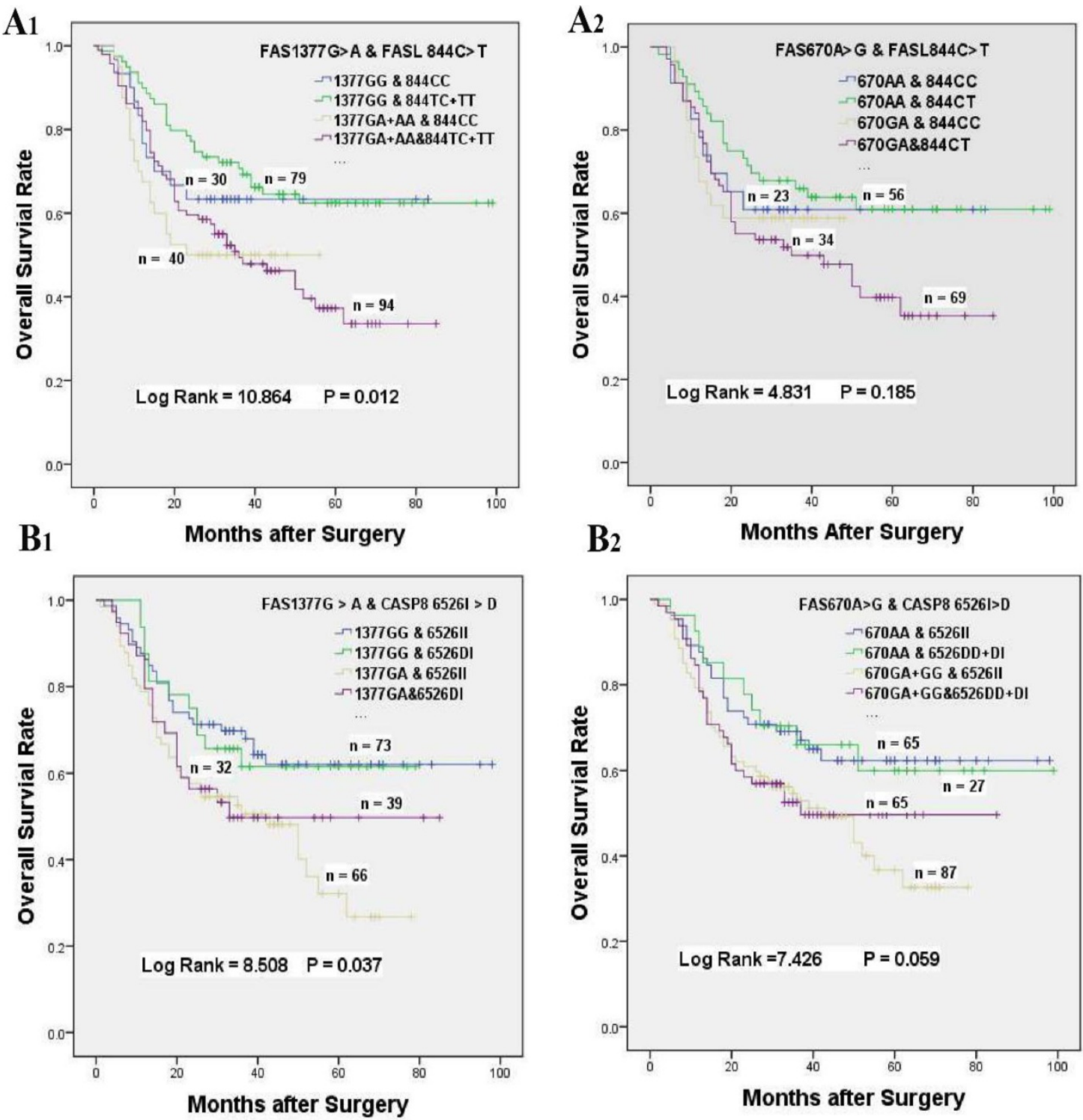

Figure 3. The effects of gene-gene interactions on the overall survival of GC patients accepted platinum-based chemotherapy. (A) Kaplan-Meier survival curves of FASL $844 \mathrm{C}$ $>$ T interaction with FAS $1377 \mathrm{G}>\mathrm{A}\left(\mathrm{A}_{1}\right)$ or FAS $670 \mathrm{~A}>\mathrm{G}\left(\mathrm{A}_{2}\right)$ on the survival of $\mathrm{GC}$ patients underwent chemotherapy. (B) Kaplan-Meier survival curves of CASP8 $6526 \mathrm{~N}$ I $>$ D interaction with FAS $1377 \mathrm{G}>\mathrm{A}\left(\mathrm{B}_{1}\right)$ or FAS $670 \mathrm{~A}>\mathrm{G}\left(\mathrm{B}_{2}\right)$ on the survival of $G C$ patients underwent chemotherapy.

The FAS 670 A > G and $1377 \mathrm{G}>\mathrm{A}$ variants could decrease Fas level and diminish Fas promoter activity, thereby down-regulating Fas-mediated apoptosis [21,35]. Previous meta-analysis has indicated that FAS $1377 \mathrm{G}>\mathrm{A}$ mutations might increase Asian sensitivity to GC, but no significant associations were identified between FAS $670 \mathrm{G}>$ A variant and GC risk $[29,36]$. Besides, the genetic polymorphisms involved in Fas-induced apoptosis pathway might also play significant effects on the prognosis of some types of cancer patients underwent platinum-based chemotherapy [19,20,37] [38]. For example, $\mathrm{Li}$ et al. indicated that the prognosis (PFS and OS) of FAS $670 \mathrm{~A}>\mathrm{G}$ polymorphism GG genotype for advanced non-small cell lung cancer
(NSCLC) patients accepted platinum-based adjuvant treatment was worse than that of GA or AA genotype [31]. Similar to those study, our results also showed that chemotherapy GC patients carrying FAS 1377 GA + AA or 670 GA + GG genotypes had poor survival compared with those wide type $[39,40]$. Stratified analyses based on multiple clinical and pathological characteristics were conducted to further identify the potential chemoresistance factors. $\mathrm{Li}$ et al. demonstrated that the advanced NSCLC patients with tumor size larger than $3 \mathrm{~cm}$ had poor response to platinum-based chemotherapy for FAS 670 A > G mutation [31]. For GC patients, our results showed that many subgroups, including age, tumor size and site, tumor differentiation, histological types, lymph node metastasis, invasion depth and TNM stage had 
significant effects on the prognosis of chemotherapy patients with FAS 1377 GA + AA or 670 GA + GG genotypes (Table 3 ). In the combination analysis, a greater than additive effect was identified when patients simultaneously carrying FAS 1377 GA and FAS 670 GA genotypes $(\mathrm{HR}=1.830,95 \% \mathrm{CI}=$ 1.179-2.840, log-rank $P<0.001)$. This may due to that both the two polymorphisms affected the gene expression level in the same causal pathway, thereby enhancing the synergistic effects [41,42].

Activation-induced cell death (AICD) referred to the apoptosis of tumor-infiltrating lymphocytes (TILs), which may lead to the escape of transformed cells to fight the killing of anti-cancer T lymphocytes, thereby promoting tumor progression $[43,44]$. The AICD of $\mathrm{T}$ lymphocytes was a FASL-dependent process and accumulated evidences have demonstrated that the elevated expression of FASL could counterattack FAS-sensitive TILs, which may lead to immune privilege and then weaken the cancer cells' sensitivity to chemotherapy [45]. But, recent researches on the relevance between FASL $844 \mathrm{C}>\mathrm{T}$ variant with the sensitivity to cancer or the chemotherapy response were controversial. For example, some studies have demonstrated that FASL $844 \mathrm{C}>\mathrm{T}$ mutation can diminish cancer development and progression $[46,47]$. Whereas, $\mathrm{Li}$ et al. showed that FASL $844 \mathrm{C}>\mathrm{T}$ genetic variant had no significant effects on chemotherapy response or survival prognosis for advanced NSCLC patients [31]. Similarly, no associations were identified for GC patients accepted platinum-based adjuvant chemotherapy in our investigation $(\mathrm{HR}=0.851,95 \% \mathrm{CI}=$ 0.561-1.290), but the protection effect may be shown if more patents were enrolled. The explanations for those phenomena were that FASL $844 \mathrm{C}>\mathrm{T}$ mutation strongly decreased FASL expression and the AICD process of tumor-specific $\mathrm{T}$ cell, which may lead to stronger immune surveillance and then enhance the sensitivity to chemotherapy [48,49]. Interestingly, although FAS 1377 GA + GG genotypes could shorten the overall survival time of chemotherapy patients, the poor survival was neutralized when patients simultaneous with FASL 844 TC + TT and FAS 1377 GA + GG genotypes. The similar neutralization was also existed when patients simultaneously carrying FASL 844 TC and FAS 670 GA genotypes (Table 4). The explanations for the above antagonistic effects may due to that the interactions between FASL and FAS could neutralize the effects of the single gene $[50,51]$.

In addition, CASP8 was also a central regulator in FAS-FASL mediated AICD of TILs, but the current researches on the associations between $6526 \mathrm{~N} \mathrm{I}>\mathrm{D}$ variation with patient's survival were debatable [52].
For no chemotherapy cohort, Frank et al. has showed that $6526 \mathrm{~N} \mathrm{D}$ was a risk factor for cancer patients [53]. However, many other researchers have revealed that $6526 \mathrm{~N}$ del could decrease the cancer risks [54,55]. For ovarian cancer patients received chemotherapy, Zhang et al. indicated that the CASP8 $6526 \mathrm{~N} \mathrm{I} \mathrm{>} \mathrm{D}$ mutation could weaken cisplatin-induced apoptosis [56]. However, Li et al. demonstrated $6526 \mathrm{~N}$ ins $>$ del variant had a better prognosis for the cutaneous melanoma patients accepted chemotherapy [57]. In our investigation, no remarkable association was identified between $6526 \mathrm{~N}$ I > D mutation with chemotherapy patient's survival. But the protection effect may be shown if a larger number of patients were enrolled in our study. This might due to $6526 \mathrm{~N} \mathrm{I}$ $>\mathrm{D}$ variation could decrease CASP8 expression and enzyme activity, which could reduce $\mathrm{T}$ lymphocytes apoptotic and then increase immune surveillance $[57,58]$. In the combination analysis, the poor survival of patients carrying FAS 670 GA + GG genotype was maintained when CASP8 6526 II genotypes coexisted at the same time. Interestingly, the inferior survival was neutralized when patients simultaneously carrying CASP8 6526 DI + DD and FAS 670 GA + GG genotypes, which were similar to the phenomenon for the patients simultaneous with FAS 1377GA and CASP8 652 6DI genotypes. Those neutralization may be caused by the interactions between genes involved in the Fas-induced apoptosis pathway $[59,60]$.

In conclusion, our findings demonstrated that FAS 670 A > G and $1377 \mathrm{G}>$ A mutations lead to a poor reaction to platinum-based adjuvant chemotherapy, which may serve as potential biomarkers to predict the chemotherapy efficacy for stage II-III GC patients. Besides, the gene-gene interactions involved in Fas-mediated apoptosis may enhance or neutralize the chemosensitivity. All in all, our findings provided a strong basis for future prospective clinical trials to choose more effective chemotherapy drugs and design effective personalized therapy regimens for GC patients.

\section{Abbreviations}

GC: Gastric Cancer; SNPs: single nucleotide polymorphisms; TNF: tumor necrosis factor; Sp1: Transcription factor Sp1; STAT1: Signal Transducer and Activator of Transcription 1; CTL: Cytotoxic T Lymphocytes; MST: Median Survival Time; HRs: Hazard Ratios; 95\%CIs: 95\% Confidence Intervals; AICD: Activation-Induced Cell Death; TILs: TumorInfiltrating Lymphocytes.

\section{Supplementary Material}

Supplementary figures and tables.

http://www.jcancer.org/v12p0849s1.pdf 


\section{Acknowledgements}

This work was partly supported by National Natural Science Foundation of China to Dr. Tingting Zhao (81800647); Postgraduate Research \& Practice Innovation Program of Jiangsu Province (KYCX20_0048) to Dr Tingting Zhao; Key Technology R\&D Program of Gansu Province (144FKCA089); Woman \& Child Health Research Program of Jiangsu Province (F201739); Zhenjiang Social Development Project (SH2018045).

\section{Author Contributions}

J.F.C. and W.S.Q. conceived and designed the experiments. T.T.Z. and W.L. performed the experiments. T.T.Z. and W.L. recruited and collected patient information and specimens. T.T.Z. analyzed the data and wrote the manuscript. All authors reviewed the manuscript.

\section{Availability of data and materials}

All of our materials, data and related protocols are available for all readers for free.

\section{Informed consent and ethics approval}

All enrolled GC patients signed informed consents to use postoperative tissues for clinical researches. All of our researches protocol was approved by the Institutional Review Committee of Nanjing Medical University.

\section{Competing Interests}

The authors have declared that no competing interest exists.

\section{References}

1. Ferlay J, Soerjomataram I, Dikshit R, Eser S, Mathers C, Rebelo M, Parkin DM, Forman D, Bray F. Cancer incidence and mortality worldwide: sources, methods and major patterns in GLOBOCAN 2012. Int J Cancer. 2015;136:E359-386

2. Torre LA, Bray F, Siegel RL, Ferlay J, Lortet-Tieulent J, Jemal A. Global cancer statistics, 2012. CA: Cancer J Clin. 2015;65:87-108

3. Zhao T, Huo X, Chen J. Genetic polymorphism of rs9564966 G > A on 13q22.1 predicts poor survival for Chinese patients with gastric cancer. Cancer Med. 2019:8:428-436.

4. Wagner AD, Grothe W, Haerting J, Kleber G, Grothey A, Fleig WE. Chemotherapy in advanced gastric cancer: a systematic review and meta-analysis based on aggregate data. J Clin Oncol. 2006;24:2903-2909.

5. Xu Z, Chen Y, Gu D, Lee NP, Sun S, Gong W, Tan Y, Luk JM, Chen J. SOD2 rs4880 CT/CC genotype predicts poor survival for Chinese gastric cancer patients received platinum and fluorouracil based adjuvant chemotherapy. Am J Transl Res. 2015;7:401-410.

6. Group G, Paoletti X, Oba K, Burzykowski T, Michiels S, Ohashi Y, Pignon JP, Rougier P, Sakamoto I, Sargent D, Sasako M, Van Cutsem E, Buyse M. Benefit of adjuvant chemotherapy for resectable gastric cancer: a meta-analysis. Jama. 2010;303:1729-1737.

7. Huang J, Zhao Y, Xu Y, Zhu Y, Huang J, Liu Y, Zhao L, Li Z, Liu H, Wang QL, Qi X. Comparative effectiveness and safety between oxaliplatin-based and cisplatin-based therapy in advanced gastric cancer: A meta-analysis of randomized controlled trials. Oncotarget. 2016; 7:34824-34831.

8. Fraser $\mathrm{M}$, Leung $\mathrm{B}$, Jahani-Asl A, Yan $\mathrm{X}$, Thompson WE, Tsang BK. Chemoresistance in human ovarian cancer: the role of apoptotic regulators. Reprod Biol Endocrinol. 2003;1:66.

9. Villa-Morales M, Fernandez-Piqueras J. Targeting the Fas/FasL signaling pathway in cancer therapy. Expert Opin Ther Targets. 2012;16:85-101.

10. Zornig M, Hueber A, Baum W, Evan G. Apoptosis regulators and their role in tumorigenesis. Biochim Biophys Acta. 2001:1551:F1-37.
11. Thompson CB. Apoptosis in the pathogenesis and treatment of disease. Science. 1995;267:1456-1462

12. Hajra KM, Liu JR. Apoptosome dysfunction in human cancer. Apoptosis. 2004;9:691-704.

13. Evan GI, Vousden KH. Proliferation, cell cycle and apoptosis in cancer. Nature. 2001;411:342-348.

14. Nagata S, Golstein P. The Fas death factor. Science. 1995;267:1449-1456.

15. Suda T, Takahashi T, Golstein P, Nagata S. Molecular cloning and expression of the Fas ligand, a novel member of the tumor necrosis factor family. Cell. 1993:75:1169-1178.

16. Poulaki V, Mitsiades CS, Mitsiades N. The role of Fas and FasL as mediators of anticancer chemotherapy. Drug Resist Updat. 2001;4:233-242.

17. Chaudhry P, Srinivasan R, Patel FD. Expression of the major fas family and Bcl-2 family of proteins in epithelial ovarian cancer (EOC) and their correlation to chemotherapeutic response and outcome. Oncol Res. 2010;18:549-559.

18. Petak I, Houghton JA. Shared pathways: death receptors and cytotoxic drugs in cancer therapy. Pathol Oncol Res. 2001;7:95-106.

19. Nagao M, Nakajima Y, Hisanaga M, Kayagaki N, Kanehiro H, Aomatsu Y, Ko S, Yagita H, Yamada T, Okumura K, Nakano H. The alteration of Fas receptor and ligand system in hepatocellular carcinomas: how do hepatoma cells escape from the host immune surveillance in vivo? Hepatology. 1999;30:413-421.

20. Shimonishi $T$, Isse $K$, Shibata F, Aburatani I, Tsuneyama K, Sabit H, Harada K, Miyazaki K, Nakanuma Y. Up-regulation of fas ligand at early stages and down-regulation of Fas at progressed stages of intrahepatic cholangiocarcinoma reflect evasion from immune surveillance. Hepatology. 2000;32:761-769.

21. Houston A, O'Connell J. The Fas signalling pathway and its role in the pathogenesis of cancer. Curr Opin Pharmacol. 2004;4:321-326.

22. Nagata S. Apoptosis by death factor. Cell. 1997:88:355-365.

23. Suda T, Okazaki T, Naito Y, Yokota T, Arai N, Ozaki S, Nakao K, Nagata S. Expression of the Fas ligand in cells of $\mathrm{T}$ cell lineage. J Immunol. 1995; 154:3806-3813.

24. Grenet J, Teitz T, Wei T, Valentine V, Kidd VJ. Structure and chromosome localization of the human CASP8 gene. Gene. 1999;226:225-232.

25. Huang QR, Morris D, Manolios N. Identification and characterization of polymorphisms in the promoter region of the human Apo-1/Fas (CD95) gene. Mol Immunol. 1997;34:577-582

26. Wu J, Metz C, Xu X, Abe R, Gibson AW, Edberg JC, Cooke J, Xie F, Cooper GS, Kimberly RP. A novel polymorphic CAAT/enhancer-binding protein beta element in the FasL gene promoter alters Fas ligand expression: a candidate background gene in African American systemic lupus erythematosus patients. J Immunol. 2003;170:132-138

27. Sun T, Gao Y, Tan W, Ma S, Shi Y, Yao J, Guo Y, Yang M, Zhang X, Zhang Q, Zeng C, Lin D. A six-nucleotide insertion-deletion polymorphism in the CASP8 promoter is associated with susceptibility to multiple cancers. Nat Genet. 2007;39:605-613.

28. Srinivasan R, T SS, Gupta A, Kaliaperumal S. Hypopyon iritis after primary fresh amniotic membrane transplantation. Cornea. 2007;26:1275-1276.

29. Wu Z, Wang H, Chu X, Chen J, Fang S. Association between FAS-1377 G/A polymorphism and susceptibility to gastric cancer: evidence from a meta-analysis. Tumour Biol. 2013;34:2147-2152.

30. El-Hamamsy M, Ghali RR, Saad AS, Shaheen SM, Salem AM. FAS and FASL genetic polymorphisms impact on clinical outcome of malignant pleural mesothelioma. Onco Targets Ther. 2016;9:6857-6863.

31. Tao KY, Li XX, Xu WZ, Wang Y, Zhu SM, Xie HX, Luo WH, Xu YJ, Xu XL. Prognostic role of apoptosis-related gene functional variants in advanced non-small-cell lung cancer patients treated with first-line platinum-based chemotherapy. Onco Targets Ther. 2015;8:147-155.

32. Lauren P. The Two Histological Main Types of Gastric Carcinoma: Diffuse and So-Called Intestinal-Type Carcinoma. An Attempt at a Histo-Clinical Classification. Acta Pathol Microbiol Scand. 1965;64:31-49.

33. Wang M, Bai J, Tan Y, Wang S, Tian Y, Gong W, Zhou Y, Gao Y, Zhou J, Zhang $Z$. Genetic variant in PSCA predicts survival of diffuse-type gastric cancer in a Chinese population. Int J Cancer. 2011;129:1207-1213.

34. Xiao HW, Lai XY, Luo Y, Shi JM, Tan YM, He JS, Xie WZ, Li L, Zhu XL, Zhu JJ, Sun J, Wei GQ, Jin L, Liu LZ, Wu KN, Yu XH, Cai Z, Lin MF, Ye XJ, Huang H. Relationship between TNFA, TNFB and TNFRII gene polymorphisms and outcome after unrelated hematopoietic cell transplantation in a Chinese population. Bone Marrow Transplant. 2011;46:400-407.

35. Mizutani $Y$, Hongo F, Sato N, Ogawa O, Yoshida O, Miki T Significance of serum soluble Fas ligand in patients with bladder carcinoma. Cancer. 2001;92:287-293.

36. Tian J, Pan F, Li J, Ma Y, Cen H, Pan HF, Pan YY, Ye DQ. Association between the FAS/FASL polymorphisms and gastric cancer risk: a meta-analysis. Asian Pac J Cancer Prev. 2012;13:945-951.

37. ElOjeimy S, McKillop JC, El-Zawahry AM, Holman DH, Liu X, Schwartz DA, Day TA, Dong JY, Norris JS. FasL gene therapy: a new therapeutic modality for head and neck cancer. Cancer Gene Ther. 2006;13:739-745.

38. Chaudhry P, Srinivasan R, Patel FD, Gopalan S, Majumdar S. Serum soluble Fas levels and prediction of response to platinum-based chemotherapy in epithelial ovarian cancer. Int J Cancer. 2008;122:1716-1721. 
39. Dutta T, Sharma H, Kumar L, Dinda AK, Kumar S, Bhatla N, Singh N. Neoadjuvant chemotherapy for epithelial ovarian cancer--role of apoptosis. Cancer Chemother Pharmacol. 2005;56:427-435.

40. Anthoney DA, Mcllwrath AJ, Gallagher WM, Edlin AR, Brown R. Microsatellite instability, apoptosis, and loss of p53 function in drug-resistant tumor cells. Cancer Res. 1996;56:1374-1381.

41. Brennan P. Gene-environment interaction and aetiology of cancer: what does it mean and how can we measure it? Carcinogenesis. 2002;23:381-387.

42. Zhang X, Miao X, Sun T, Tan W, Qu S, Xiong P, Zhou Y, Lin D. Functional polymorphisms in cell death pathway genes FAS and FASL contribute to risk of lung cancer. J Med Genet. 2005;42:479-484.

43. Green DR, Droin N, Pinkoski M. Activation-induced cell death in T cells. Immunol Rev. 2003;193:70-81.

44. Maher S, Toomey D, Condron C, Bouchier-Hayes D. Activation-induced cell death: the controversial role of Fas and Fas ligand in immune privilege and tumour counterattack. Immunol Cell Biol. 2002;80:131-137.

45. O'Connell J, Bennett MW, O'Sullivan GC, Collins JK, Shanahan F. The Fas counterattack: cancer as a site of immune privilege. Immunol Today. 1999;20:46-52.

46. O'Connell J, O'Sullivan GC, Collins JK, Shanahan F. The Fas counterattack: Fas-mediated $\mathrm{T}$ cell killing by colon cancer cells expressing Fas ligand. J Exp Med. 1996;184:1075-1082.

47. Koyama S, Koike N, Adachi S. Fas receptor counterattack against tumor-infiltrating lymphocytes in vivo as a mechanism of immune escape in gastric carcinoma. J Cancer Res Clin Oncol. 2001;127:20-26.

48. Bennett MW, O'Connell J, O'Sullivan G C, Roche D, Brady C, Kelly J, Collins JK, Shanahan F. Expression of Fas ligand by human gastric adenocarcinomas: a potential mechanism of immune escape in stomach cancer. Gut. 1999;44:156-162.

49. Huber V, Fais S, Iero M, Lugini L, Canese P, Squarcina P, Zaccheddu A, Colone M, Arancia G, Gentile M, Seregni E, Valenti R, Ballabio G, Belli F, Leo E, Parmiani G, Rivoltini L. Human colorectal cancer cells induce T-cell death through release of proapoptotic microvesicles: role in immune escape. Gastroenterology. 2005;128:1796-1804.

50. Sun T, Miao X, Zhang X, Tan W, Xiong P, Lin D. Polymorphisms of death pathway genes FAS and FASL in esophageal squamous-cell carcinoma. J Natl Cancer Inst. 2004;96:1030-1036.

51. Lai HC, Lin WY, Lin YW, Chang CC, Yu MH, Chen CC, Chu TY, Genetic polymorphisms of FAS and FASL (CD95/CD95L) genes in cervical carcinogenesis: An analysis of haplotype and gene-gene interaction. Gynecol Oncol. 2005;99:113-118.

52. Ho PK, Hawkins CJ. Mammalian initiator apoptotic caspases. FEBS J. 2005;272:5436-5453.

53. Frank B, Rigas SH, Bermejo JL, Wiestler M, Wagner K, Hemminki K, Reed MW, Sutter C, Wappenschmidt B, Balasubramanian SP, Meindl A, Kiechle M, Bugert P, Schmutzler RK, Bartram CR, Justenhoven C, Ko YD, Bruning T, Brauch H, Hamann U, Pharoah PP, Dunning AM, Pooley KA, Easton DF, Cox A, Burwinkel B. The CASP8 $-6526 \mathrm{~N}$ del promoter polymorphism and breast cancer risk: a multicenter study. Breast Cancer Res Treat. 2008;111:139-144.

54. Son JW, Kang HK, Chae MH, Choi JE, Park JM, Lee WK, Kim CH, Kim DS, Kam S, Kang YM, Park JY. Polymorphisms in the caspase-8 gene and the risk of lung cancer. Cancer Genet Cytogenet. 2006;169:121-127.

55. Lubahn J, Berndt SI, Jin CH, Klim A, Luly J, Wu WS, Isaacs S, Wiley K, Isaacs WB, Suarez BK, Hayes RB, Kibel AS. Association of CASP8 D302H polymorphism with reduced risk of aggressive prostate carcinoma. Prostate. 2010;70:646-653

56. Zhang X, Wang X, Song X, Liu C, Shi Y, Wang Y, Afonja O, Ma C, Chen YH, Zhang L. Programmed cell death 4 enhances chemosensitivity of ovarian cancer cells by activating death receptor pathway in vitro and in vivo. Cancer Sci. 2010;101:2163-2170

57. Li C, Zhao H, Hu Z, Liu Z, Wang LE, Gershenwald JE, Prieto VG, Lee JE, Duvic M, Grimm EA, Wei Q. Genetic variants and haplotypes of the caspase-8 and caspase-10 genes contribute to susceptibility to cutaneous melanoma. Hum Mutat. 2008;29:1443-1451.

58. Yang M, Sun T, Wang L, Yu D, Zhang X, Miao X, Liu J, Zhao D, Li H, Tan W, Lin D. Functional variants in cell death pathway genes and risk of pancreatic cancer. Clin Cancer Res. 2008;14:3230-3236.

59. Zhao T, Xu Z, Gu D, Wu P, Huo X, Wei X, Tang Y, Gong W, He ML, Chen J. The effects of genomic polymorphisms in one-carbon metabolism pathways on survival of gastric cancer patients received fluorouracil-based adjuvant therapy. Sci Rep. 2016;6:28019.

60. Kim DH, Sriharsha L, Xu W, Kamel-Reid S, Liu X, Siminovitch K, Messner HA, Lipton JH. Clinical relevance of a pharmacogenetic approach using multiple candidate genes to predict response and resistance to imatinib therapy in chronic myeloid leukemia. Clin Cancer Res. 2009;15:4750-4758. 